\title{
La brecha de género en el acceso a la tierra: una mirada desde la política agropecuaria dirigida a las mujeres rurales en Costa Rica
}

\author{
Gender gap in access to land: a view from the agricultural policy \\ aimed at rural women in Costa Rica
}

\author{
Marylaura Acuña Alvarado \\ Universidad Católica de Lovaina \\ Lovaina, Bélgica \\ marylaura.acunaalvarado@gmail.com
}

\begin{abstract}
RESUMEN. El artículo presenta una reflexión en torno a la brecha de género en el acceso a la tierra en Costa Rica, mediante el estudio de las principales políticas públicas del sector agropecuario dirigidas hacia las mujeres. Desde la perspectiva de la economía feminista, el estudio busca resaltar la importancia de la tenencia, uso y control de la tierra para las mujeres rurales. A partir del análisis de datos estadísticos y elementos discursivos, se plantea que de 1950 a 1990 las mujeres fueron incluidas subordinadamente al sector agropecuario, principalmente desde su rol de madres y esposas. Posteriormente, en el marco del enfoque de desarrollo sostenible promovido desde los noventas, las mujeres han tomado un rol protagónico frente a la política pública. Sin embargo, se argumenta que su participación se ha ampliado en espacios que han sido paulatinamente desmantelados por el modelo de reconversión productiva. A modo de conclusión, el artículo resalta algunos de los desafíos más apremiantes para conciliar efectivamente los ideales planteados por el discurso del desarrollo sostenible y el acceso de las mujeres a la tierra.
\end{abstract}

PALABRAS CLAVE. Brecha de género, desarrollo sostenible, política agropecuaria, tierra, mujeres

Este es un artículo de acceso abierto publicado bajo los términos de la Licencia Creative Commons 4.0 Internacional Atribución-NoComercial-SinDerivadas. 


\begin{abstract}
The article presents a reflection on the gender gap in access to land in Costa Rica, through the study of the main public policies of the agricultural sector directed towards women. From the perspective of feminist economics, the study seeks to highlight the importance of land tenure, use and control for rural women. Based on analysis of statistical data and discursive elements, it is stated that from 1950 to 1990 women were included subordinately to the agricultural sector, mainly from their role as mothers and wives. Subsequently, within the framework of the sustainable development approach promoted since the 1990s, women have taken a leading role in public policy. However, it is argued that their participation has expanded in spaces that have been gradually dismantled by the productive reconversion model. In conclusion, the article highlights some of the most pressing challenges to effectively reconcile the ideals posed by the discourse of sustainable development and women's access to land.
\end{abstract}

KEYWORDS. Gender gap, sustainable development, agricultural policy, land, women

\title{
INTRODUCCIÓN
}

Históricamente, la distribución desigual de la tierra ha sido un elemento conflictivo en América Latina. Si bien entre 1950 y 1980 la mayoría de los gobiernos latinoamericanos incurrieron en reformas redistributivas, en muchas ocasiones las mujeres no se beneficiaron de dichos procesos. Las mujeres fueron directamente excluidas de las reformas agrarias o bien, fueron incluidas de forma subordinada. Es decir, incorporadas desde sus roles de madres, esposas e hijas, limitando su acceso a la tierra según su relación con alguna figura masculina del núcleo familiar (Castillo, 2015). Dichos mecanismos de inclusión y exclusión de la política pública constituyen algunos de los factores institucionales que contribuyen a la brecha de género en el acceso a la tierra. Otros factores son de carácter estructural e ideológico-cultural, como por ejemplo las tradiciones ligadas a la herencia de la tierra a nivel familiar, el reconocimiento social de las mujeres como productoras y sesgos de género en el mercado de tierras (Lastarria-Cornhiel et al., 2011).

A partir de la década de los ochentas, los movimientos de mujeres hicieron eco en la escena de las conferencias y organismos internacionales. Principalmente, el discurso del desarrollo sostenible destacó el papel crucial de las mujeres en cuanto a las economías rurales, la seguridad alimentaria y la protección de los recursos 
naturales. En dicho contexto, diversos países incurrieron en medidas institucionales para reconocer los derechos de las mujeres en cuanto al acceso a la tierra y a la vez mejorar sus condiciones de vida. Sin embargo, dicho avance en materia de derechos ocurrió de forma paralela a un proceso de desmantelamiento de la función social del Estado, enmarcado en la firma de los Planes de Ajuste Estructural (PAEs) en América Latina. El proceso de reconversión de la matriz productiva implicó un cambio en el modelo de dotación de tierras y apoyo a la producción, en detrimento de la agricultura de subsistencia. Al ser este tipo de agricultura en donde las mujeres tienen un mayor aporte, ellas son afectadas diferenciadamente por los cambios en la estructura de tenencia de la tierra (Korol, 2016).

Actualmente, en América Latina, en promedio solamente un $18 \%$ de las explotaciones agrícolas están a cargo de mujeres. Además, solo un $10 \%$ del total de créditos y un $5 \%$ del total de asistencia técnica son recibidos por ellas. Sin embargo, las mujeres juegan un papel crucial en la producción de alimentos en los países latinoamericanos (Organización de las Naciones Unidas [ONU], 2015). Ellas realizan una gran cantidad de labores que usualmente no son contabilizadas en las estadísticas económicas o agropecuarias. Las mujeres rurales, de origen campesino, indígena y afrodescendiente, desempeñan diversas labores como agricultoras, pescadoras, recolectoras y trabajadoras agrícolas. Asimismo, realizan dobles y hasta triples jornadas de trabajo en las huertas familiares, cuido de los animales, preparación de los alimentos, recolección de agua, cuidado de infantes, personas enfermas y adultas mayores, y demás labores de cuido. A pesar de que su participación es crucial en el desarrollo rural, sufren de diversas dificultades para acceder a la tierra, y cuando la tienen, suele ser de menor extensión y de menor calidad. Asimismo, tienen limitantes para acceder a insumos y crédito para trabajar la tierra, así como para tomar decisiones sobre ella (Food and Agriculture Organization [FAO], 2011).

Para entender la brecha de género en el acceso a la tierra, es necesario estudiar los diferentes factores que afectan a las mujeres rurales. Es decir, los espacios donde se generan las desigualdades y las resistencias, tales como el Estado, el mercado, la familia y la comunidad. Este estudio se concentra en analizar el rol de las instituciones estatales en cuanto a la tenencia, uso y control de las mujeres sobre la tierra, con el fin de develar algunos de los mecanismos 
históricos de exclusión e inclusión de las mujeres a la política pública en materia agropecuaria. El artículo plantea que, si bien en los últimos treinta años ha habido un cambio significativo en cuanto a la posición de las mujeres en el sector, esta transformación ha sido principalmente discursiva. Es decir, si bien las mujeres han sido mayormente visibilizadas en el desarrollo sostenible, narrativa crucial de la gobernanza ambiental en Costa Rica, persiste un modelo de distribución de la tierra sumamente desigual, el cual les impide mejorar sus condiciones de vida.

El texto se estructura de manera que, en una primera parte, se disponen algunos datos estadísticos que permiten introducir la problemática de la brecha de género en el acceso a la tierra en Costa Rica. En una segunda parte, se presenta un análisis del rol de las mujeres frente a la política pública en materia agropecuaria, principalmente en cuanto a los modelos de dotación de tierras. El período de estudio está dividido en dos momentos, el primero va de 1950 a 1990 y el segundo de 1990 hasta la actualidad. Dicha división responde principalmente a la promulgación de la Ley de Promoción de Igualdad Social de la Mujer en 1990, la cual representó un hito importante que, junto a otras acciones, significa un cambio crucial en cuanto a los derechos de las mujeres sobre la tierra. Más adelante, como tercera parte, el artículo argumenta sobre los principales desafíos institucionales en cuanto a la tierra para las mujeres.

El artículo es producto de una investigación realizada en el marco de un trabajo final de graduación. Como parte de la metodología del estudio se realizaron entrevistas semiestructuradas a funcionarios y funcionarias encargadas del tema de género en el seno de la Red Sectorial de Género del Sector Agropecuario. Esto se hizo con el fin de conocer a profundidad la estructura organizativa respecto al tema de género, así como algunos avances y retos generales. Concretamente se entrevistó a los enlaces de género del Ministerio de Ganadería y Agricultura (MAG), el Instituto de Desarrollo Rural (INDER), la Secretaría Ejecutiva de Planificación Sectorial Agropecuaria (SEPSA) y la persona directora de la Fundación Nacional Clubes 4 S del MAG. Las entrevistas se desarrollaron durante mayo y abril del 2018. En cuanto a fuentes secundarias, se consultaron diferentes fuentes bibliográficas incluyendo artículos e investigaciones académicas, documentación institucional (políticas nacionales, planes sectoriales, reportes, memorias) y archivos estadísticos nacionales. 


\section{DEFINIR, MEDIR Y CONTEXTUALIZAR LA BRECHA DE GÉNERO}

\section{Insumos desde la economía feminista para definir la relación mujer-tierra}

La brecha de género en el acceso a la tierra es definida como la desigualdad entre hombres y mujeres en el disfrute pleno de derechos relativos a la tenencia, uso y control de la propiedad. Este estudio se interesa por el acceso a la propiedad con fines agropecuarios, lo cual incluye también una serie de activos relacionados a la tierra, tales como semillas, ganado, trabajo, educación, servicios básicos, asistencia financiera y tecnológica (FAO, 2011). Al hablar de derecho sobre la tierra, en realidad, se hace referencia a un conjunto de derechos que dependen entre sí. En primer lugar, el derecho de uso, el cual se refiere a utilizar la tierra para fines productivos, vivir en ella o desarrollar otras actividades diarias. En segundo lugar, el derecho de control, es decir, la capacidad de tomar decisiones sobre los usos de la tierra, la obtención de ganancias económicas y cómo éstas se distribuyen o se gastan. En tercer lugar, el derecho de transferencia, referente al poder de transferir o reasignar los derechos de uso y de control de la tierra (Fuentes, 2010).

Además de un reconocimiento legal de derechos, el acceso a la tierra comprende también la legitimación social de estos derechos. Es decir, el reconocimiento de la sociedad sobre la autonomía de las mujeres para tomar decisiones sobre la tierra (Agarwal, 1994). Dicha legitimación resulta esencial al entender la brecha de género, ya que a pesar de que la legislación ha evolucionado para garantizar igualdad ante la ley, las mujeres encuentran numerosas limitantes para efectivamente adquirir, utilizar y controlar sus predios. En otras palabras, la igualdad formal en el derecho de propiedad no ha resultado en una igualdad real en la distribución de dicha propiedad. Esta desigualdad está relacionada con factores en la familia, la comunidad, el mercado económico y el Estado. A lo interno de estas dimensiones, existen mecanismos de exclusión e inclusión de carácter legal, cultural, estructural e institucional que perpetúan las condiciones desfavorables de las mujeres frente a la tierra (Deere y León, 2000).

El riesgo de vivir en condiciones de pobreza y el bienestar físico 
de una mujer rural y de su familia dependen significativamente del acceso a bienes productivos tales como la tierra y el conjunto de activos ligados a ella (FAO, 2011). Asimismo, el acceso a la tierra puede influir en el poder de negociación de las mujeres. Desde la perspectiva de la economía feminista, dicho poder es definido como fall-back position (posición de resguardo) de una mujer. Esta posición se refiere a la capacidad de acceder a un sustento, patrimonial o social, que les permita a las mujeres ciertos grados de autonomía para deshacer un matrimonio disfuncional, actuar ante situaciones de violencia doméstica o mejorar sus condiciones de vida, como algunos ejemplos. La posición de resguardo de una mujer en el ámbito rural está mayoritariamente relacionada con los siguientes elementos: la propiedad y el control de los bienes económicos, principalmente la tierra; el acceso al empleo o bien otras formas de generación de ingresos; el acceso a los recursos comunales (por ejemplo, los bosques o los terrenos comunitarios); el acceso al apoyo social externo (contar con redes de apoyo); y finalmente, el acceso a apoyo estatal o de programas no gubernamentales (Agarwal, 1997).

Más allá de los dichos elementos, la tierra puede constituir un medio para fomentar la organización comunitaria de las mujeres y de esta manera acceder a recursos productivos y educativos o participar activamente en política local (Ardito y Bórquez, 2009). Esto resulta evidente al observar la creciente participación de las mujeres en movimientos comunitarios, regionales y nacionales. Desde el movimiento campesino, las mujeres han alzado la voz en contra de los megaproyectos de la agroindustria, como los transgénicos y los monocultivos. Desde las luchas indígenas, las mujeres han tomado protagonismo en la defensa del territorio. Finalmente, desde las organizaciones ambientales, las mujeres han tomado un papel importante en resistencia contra los proyectos de extractivismo, por medio de acciones legales, protestas, entre otras (Guillamón y Ruíz, 2015).

\section{Medir la brecha de género: lo cuantitativo y lo cualitativo}

La brecha de género en cuanto a la tierra es definida como la diferencia observada entre hombres y mujeres en cuanto al acceso, uso y tenencia de la propiedad agropecuaria. Debido a que esta definición incluye dimensiones no solo cuantitativas sino también cua- 
litativas, resulta difícil de medir, describir y analizar. En América Latina, los censos agropecuarios han profundizado relativamente poco sobre la brecha, debido a una carencia de enfoque de género en la formulación, aplicación y análisis de los instrumentos estadísticos. Por su parte, las encuestas de uso del tiempo han mostrado datos significativos en cuanto a las desigualdades en la carga de trabajo entre hombres y mujeres, pero sin entrar en mayor detalle sobre la propiedad de la tierra.

En Costa Rica, la principal fuente para la medición de la brecha de género en el acceso a la tierra es el Censo Nacional Agropecuario (CENAGRO). Según dicho instrumento, para el 2015 se registró que de 80972 personas productoras ${ }^{1}$, solamente 12598 son mujeres, lo que equivale al $15,6 \%$ del total. Además, del total de títulos de propiedad con fines agropecuarios, únicamente el 8,1\% de ellos están a nombre de mujeres (Instituto Nacional de Estadística y Censos de Costa Rica [INEC], 2015). En cuanto a la extensión de las fincas agropecuarias, un $25 \%$ de las mujeres productoras tiene fincas de menos 1 hectárea de extensión, mientras que tan solo un $5 \%$ tiene 5 hectáreas o más. De manera que, el total de fincas lideradas por mujeres productoras representa tan solo un $4,4 \%$ de la extensión total de hectáreas de las fincas registradas en el país (Morales y Segura, 2016).

Si bien dicha información resulta un insumo importante para visibilizar la brecha de género, los datos presentan algunas limitantes. En primer lugar, si bien la cifra de personas productoras permite visibilizar la alarmante brecha de género, podría a su vez estar subestimando la cantidad real de mujeres productoras. Esto ocurre debido a que el Censo no contempla la posibilidad de que exista más de una persona agricultora principal por cada finca, lo cual deja abierto el paso para sesgos de género, a lo interno de las familias, al responder a la pregunta sobre la jefatura de la producción. En segundo lugar, el Censo enfoca más su interés en obtener información sobre agroindustrias y agronegocios en las unidades productivas, sin explorar sobre la situación de los hogares de las familias productoras. En la mayoría de las variables, únicamente es posible conocer sobre la situación de las mujeres que se reconocen como productoras y no de la totalidad de mujeres del hogar que

1 Esta cifra se cuenta sobre la cantidad de fincas productoras inscritas a nombre de personas físicas, no personas jurídicas. 
también tienen participación en la finca. Precisamente este grupo de mujeres duplican aquellas reconocidas como productoras (Golen y Medina, 2017).

Ambos sesgos son una tendencia en común entre los censos agropecuarios en América Latina, los cuales en muchas ocasiones carecen de enfoque de género para su formulación. A pesar de dichas limitantes, un esfuerzo importante que realiza el Censo es contemplar espacios productivos que no sean fincas, es decir, unidades agropecuarias más pequeñas. Al incluir dichos espacios, se hace visible el aporte de las mujeres a la producción, ya que 54\% de ellos están a cargo de productoras (INEC, 2015). De igual manera, este dato corresponde con la tendencia en cuanto a la tenencia de tierra en manos de mujeres a nivel global, según la cual las mujeres tienden a tener acceso mayoritariamente a espacios productivos más pequeños que fincas, como por ejemplo huertas, jardines y corrales con animales para el consumo propio del hogar. La participación de las mujeres en dichos espacios está relacionada con la desigual distribución del trabajo doméstico y el acceso a insumos para trabajar la tierra, lo cual les dificulta hacerse cargo de espacios productivos de mayor extensión (Deere y León, 2000).

Una deficiencia que presenta el censo agropecuario es que permite conocer la brecha en términos de tenencia de la tierra, con algunos datos sobre el uso, pero relativamente nula información sobre el control. Es decir, la forma en la que se toman las decisiones sobre lo que se siembra, cómo se siembra, cómo se comercializa y en general, cómo ocurre la toma de decisiones sobre el predio. Otro aspecto importante es la frecuencia de los censos y su relación con la disponibilidad de información para la hechura de políticas públicas. El último censo agropecuario a nivel nacional fue realizado en 1984 y además no presenta la misma calidad de datos en cuanto a género, impidiendo hacer un análisis de la evolución de la brecha de género en el tiempo. Una fuente alternativa para medir la brecha es la asignación de tierras a cargo del ITCO-IDA-INDER. Dichos datos resultan importantes porque permiten develar el rol de las instituciones en la distribución de la tierra para las mujeres, algunos de los cuales son presentados más adelante en el texto.

\section{Contextualizar la brecha de género: una necesidad para profundizar}


Después de apuntar hacia una definición y medición de la brecha de género, un tercer elemento esencial para estudiar el acceso de las mujeres a la tierra es ubicar los datos en los esquemas más amplios de distribución de la tierra. A partir del CENAGRO 2015 se vislumbran algunas tendencias que pueden afectar particularmente el acceso a la tierra para las mujeres. En primer lugar, cabe resaltar que se hizo notoria una reducción de la extensión agropecuaria nacional, en un 21,6\% menos que en 1984. Además, actualmente la producción agropecuaria está concentrada en pocas fincas de gran extensión (más de 500 hectáreas), como reflejo de una creciente desigualdad en la tenencia de la tierra (Morales y Segura, 2016).

El promedio de hectáreas por finca a cargo de mujeres productoras es de 8,5ha, lo que revela que las mujeres se dedican mayoritariamente a la producción de alimentos a pequeña escala. Además, se puede inferir que la producción de estas fincas no es masiva y que no necesariamente tiene altos rendimientos agrícolas como otros agronegocios de mayor extensión. También, se evidencia que las mujeres tienen una alta participación en la comercialización en el mercado local y mucho menor en el sector de la agroindustria. En cuanto a los cultivos destinados para este espacio local, los datos muestran que las mujeres hacen un menor uso de agroquímicos y consecuentemente, un mayor uso de fertilizantes orgánicos en comparación con los hombres ${ }^{2}$ (Golen y Medina, 2017).

Entre 1987 y 1995, las familias campesinas pasaron de representar el $20,7 \%$ del total de trabajadores del campo a $11,5 \%$. Esto se tradujo en una desaparición de pequeños productores, un crecimiento del trabajo temporal por parte de agricultores que, a pesar de que mantienen sus fincas, obtienen su principal ingreso a partir de su trabajo fuera de la parcela. Esta tendencia está estrechamente relacionada con la feminización de la fuerza de trabajo en las actividades agrícolas de exportación, tales como frutas y plantas ornamentales (Román, 1997). Dicha reducción en la cantidad de familias campesinas se refleja en los años posteriores en la variación porcentual de la cantidad de hectáreas dedicadas a los principales cultivos. Entre 1994 y 2013 se visualiza un aumento del cultivo de

2 Esta diferencia se presenta únicamente en los productos de consumo locales tales como banano, plátano, maíz y frijol. En cuanto a los cultivos de alta comercialización y de exportación tales como el café y la palma aceitera, la utilización de fertilizantes es igualmente alta tanto en hombres como en mujeres (Golen y Medina, 2017). 
productos no tradicionales tales como palmito, naranja, jengibre y piña, una contracción relativa en la exportación de productos tradicionales como cacao, café, caña de azúcar, banano y ganadería, y una drástica disminución de granos básicos para el consumo nacional, principalmente maíz y frijoles (León, 2015). Esto se ilustra en la Tabla 1.

Tabla 1. Cantidad de hectáreas y variación porcentual de principales cultivos, Costa Rica (1994-2013)

\begin{tabular}{cccc}
\hline Cultivo & $\mathbf{1 9 9 4}(\mathbf{h a})$ & $\mathbf{2 0 1 3}(\mathbf{h a})$ & $\begin{array}{c}\text { Variación }(\%) \\
\mathbf{1 9 9 4} \mathbf{2 0 1 3}\end{array}$ \\
\hline Arroz & 44,783 & 59,298 & +32.4 \\
Banano & 52,707 & 42,841 & -18.7 \\
Cacao & 12,000 & 4,660 & -163.5 \\
Café & 108,966 & 93,774 & -13.9 \\
Caña de azúcar & 39,800 & 63,316 & +159.0 \\
Frijol & 56,856 & 20,724 & -63.5 \\
Maíz & 18,109 & 5,978 & -67.0 \\
Naranja & 22,250 & 21,000 & -5.6 \\
Palma Africana & 26,652 & 74,512 & +279.6 \\
Piña & 7,000 & 45,000 & +642.9 \\
\hline
\end{tabular}

Fuente: Elaboración propia con base en Programa Estado de la Nación (2013)

De manera que, al estudiar la brecha de género en el acceso a la tierra es necesario tomar en cuenta otros datos que permitan dar cuenta de los esquemas de distribución de tierra. En este sentido, la información censal permite respaldar la idea de que Costa Rica ha sufrido una profunda transformación productiva en detrimento de la agricultura de granos básicos. El panorama actual revela que, en los últimos treinta años, se ha reducido considerablemente la tierra dedicada a la producción de alimentos y que la tierra está cada vez más concentrada en pocas manos. Consecuentemente, estamos ante una asalarización de las familias campesinas producto de un despojo sistemático de sus medios de producción, razón por la cual deben vender su fuerza de trabajo. Esta asalarización ha sido particularmente alta en las mujeres, quienes ocupan empleos tempo- 
rales en las agroindustrias bajo condiciones de explotación además marcadas por el género. En este contexto, resulta difícil pensar en mayores oportunidades para las mujeres en el agro. Sin embargo, los discursos en torno al desarrollo sostenible han pretendido conciliar el desmantelamiento de la agricultura campesina, la producción a gran escala, la protección del ambiente y la inclusión de las mujeres al agro.

\section{LAS MUJERES FRENTE A LOS MODELOS DE DOTACIÓN DE TIERRAS}

En esta sección se destacan las principales acciones institucionales respecto a las mujeres rurales en el sector agropecuario, haciendo énfasis en los modelos de distribución de tierras. En este sentido, se hace hincapié en la institución encargada de este tema, el ITCO-IDA-INDER, pero también se mencionan otras instancias del sector. Para el período de 1950 hasta 1990, se vislumbra una inclusión subordinada de las mujeres a las reformas de redistribución de la tierra. A partir de 1990 se generan cambios importantes en cuanto a los derechos de las mujeres, tanto a nivel legal como a nivel discursivo. En los últimos diez años, si bien la política pública ha continuado visibilizando la importancia de incluir a las mujeres en el desarrollo, existen diversos retos que afectan directamente la brecha de género. La Tabla 2 presenta un resumen de los cambios en cuanto al modelo de dotación de tierras y la posición que han ocupado las mujeres frente a la política pública en esta materia.

Tabla 2. Cambios en el modelo de dotación de tierras e inclusión de las mujeres (1949-2012)

\begin{tabular}{lccc}
\hline & \multicolumn{3}{c}{ Cambios institucionales } \\
\hline MAI* (1949) e & IDA (1982) & INDER (2012) \\
\cline { 2 - 4 } Enfoque (1961) & $\begin{array}{c}\text { Pacificación del } \\
\text { agro y Coloni- } \\
\text { zación de tierras }\end{array}$ & $\begin{array}{c}\text { Desarrollo Rural } \\
\text { Integrado (1980s) } \\
\text { y Desarrollo } \\
\text { Sostenible (1990s) }\end{array}$ & $\begin{array}{c}\text { Desarrollo Ter- } \\
\text { ritorial y Nueva } \\
\text { Ruralidad }\end{array}$
\end{tabular}




\begin{tabular}{cccc}
$\begin{array}{c}\text { Modelo de } \\
\text { dotación de tierra }\end{array}$ & $\begin{array}{c}\text { Formación de co- } \\
\text { lonias campesinas }\end{array}$ & $\begin{array}{c}\text { Formación de } \\
\text { asentamientos } \\
\text { campesinos }\end{array}$ & $\begin{array}{c}\text { Proyectos } \\
\text { productivos y } \\
\text { arrendamientos }\end{array}$ \\
$\begin{array}{c}\text { Sujeto de la adju- } \\
\text { dicación }\end{array}$ & $\begin{array}{c}\text { Unidad produc- } \\
\text { tiva familiar a } \\
\text { través de la figura } \\
\text { de jefatura de } \\
\text { hogar }\end{array}$ & $\begin{array}{c}\text { Cooperativas y } \\
\text { Unidad producti- } \\
\text { va familiar (titu- } \\
\text { lación conjunta a } \\
\text { partir de 1990) }\end{array}$ & $\begin{array}{c}\text { Titulación con- } \\
\text { junta en unidad } \\
\text { productiva } \\
\text { familiar, Cooper- } \\
\text { ativas y Grupos } \\
\text { Organizados }\end{array}$ \\
$\begin{array}{c}\text { Rol de las } \\
\text { mujeres a nivel } \\
\text { discursivo }\end{array}$ & $\begin{array}{c}\text { "Amas de casa", } \\
\text { "esposas"y } \\
\text { "ayudantes" }\end{array}$ & $\begin{array}{c}\text { Mujeres como } \\
\text { protagonistas } \\
\text { del desarrollo } \\
\text { sostenible }\end{array}$ & $\begin{array}{c}\text { Actores sociales } \\
\text { en los territorios }\end{array}$ \\
$\begin{array}{c}\text { Acciones dirigi- } \\
\text { das hacia las } \\
\text { mujeres }\end{array}$ & $\begin{array}{c}\text { Mrojoramas de } \\
\text { Hogmiento del }\end{array}$ & $\begin{array}{c}\text { Oficinas y planes } \\
\text { sectoriales }\end{array}$ & $\begin{array}{c}\text { Planificación } \\
\text { sectorial de géne- } \\
\text { ro y Equidad e } \\
\text { inclusión }\end{array}$ \\
\hline
\end{tabular}

Fuente: Baldizán y Cruz (1987); INDER (2018).

*Ministerio de Agricultura e Industria, ahora MAG.

\section{Las mujeres rurales como amas de casa (1950-1990)}

Las reformas agrarias latinoamericanas fueron impulsadas en gran medida por los intereses geoestratégicos de Estados Unidos mediante la Alianza del Progreso. La asistencia estadounidense para que los países incursionaran en programas de distribución de la tierra estaba ligado a la idea de que los movimientos revolucionarios se podrían desmovilizar si el problema de la desigualdad y la pobreza rural eran atendidos. Asimismo, la reforma pretendía aumentar la productividad de la tierra y de forma paulatina modernizar la agricultura, mediante el fortalecimiento de la clase campesina con tierra (Kay, 1995). En Costa Rica, mediante la creación del Instituto de Tierras y Colonización (ITCO) en 1961, el incipiente Estado Desarrollista buscó institucionalizar el proceso de colonización de tierras como forma de atender los conflictos ocasionados por la presión generada a causa de la expansión de la frontera agrícola (Inder, 2018).

El proceso de distribución de la tierra fue pensado para beneficiar a las familias de campesinas siguiendo la lógica de la "teoría 
del goteo", la cual asumía que las mujeres e hijos se benefician automáticamente a partir de los recursos otorgados exclusivamente a los jefes de hogar (Moser, 1999). En este sentido, la inclusión de las mujeres al desarrollo agropecuario antes de los noventas fue desde una posición predominantemente subordinada. El acceso de las mujeres a la tierra estuvo por mucho tiempo anclado a su relación con las figuras masculinas de sus núcleos familiares, ya fuese por estado civil, conyugalidad o maternidad, tanto a nivel legal como socio-cultural. Dicha inclusión subordinada se evidencia en cuatro aspectos relacionados con la tenencia, uso y control de la tierra: las adjudicaciones de tierra, el acceso a servicios de extensión agropecuaria, la participación en cooperativas agrícolas y la participación en la fuerza laboral.

\section{Adjudicación de la tierra}

Si bien actualmente las mujeres tienen igualdad ante la ley en cuanto al acceso a la tierra, esto no siempre fue así. La ley de reforma agraria no fue neutral al género y su articulado lo hizo evidente. La mayor parte del texto de la ley está escrito bajo el concepto de una unidad productiva familiar que asume en el hombre la jefatura del hogar y en la esposa la beneficiaria secundaria de la tierra. Por ejemplo, el Artículo 68 indica la posibilidad de adjudicación de una parcela a "la esposa" del parcelero, en caso del abandono injustificado de éste. Sin embargo, esto solo bajo la condición de que la mujer cumpliese con una serie de criterios asociados a la capacidad técnica y experiencia previa en trabajos agrarios (Ley de Tierras y Colonización (ITCO INDER), 1961, Art. 62). El sistema de puntaje para seleccionar a los potenciales beneficiarios resultó ser más favorable para los jefes de hogar, padres de más de un hijo y con experiencia agrícola, en detrimento de las mujeres (Deere y León, 2000).

En la práctica institucional, la aplicación de esta normativa se tradujo en una discriminación sistemática de las mujeres a las parcelas otorgadas por el entonces ITCO. Esto se evidencia en el hecho de que entre 1963 y 1988, de las personas beneficiadas por la asignación de tierras en asentamientos campesinos tan solo un 13,39\% fueron mujeres, lo que representa un $19,55 \%$ del total de hectáreas 
adjudicadas (Madden, 1992). En la dinámica familiar, la ley abonó a la construcción del ideario de la familia campesina tradicional, entendida como un modelo biparental en donde la posición del jefe de hogar estaba asociada de forma casi indiscutible al hombre, quien es considerado como el representante de los intereses de toda la familia y la persona encargada del manejo de los recursos familiares (Blanco, 1997).

\section{Extensión agropecuaria}

En cuanto al acceso a los programas de extensión agropecuaria, la práctica institucional se basó también en una división sexual del trabajo. Desde 1949 se creó el programa Clubes 4S y Amas de Casa, en el seno del entonces Ministerio de Agricultura e Industria, con el objetivo de promover el desarrollo de las fincas, los hogares y la comunidad rural. Mediante este proyecto, los hombres parceleros recibían capacitación y recursos técnico-financieros para el trabajo de la tierra, mientras que las mujeres recibían talleres de manualidades, mejoramiento del hogar y nutrición aplicada. Los clubes de mujeres tenían menos apoyo financiero, menos estructuración y menos capacidad operativa en el seno de la institución, en comparación con otros proyectos (Baldizán y Cruz, 1987).

\section{Participación en cooperativas}

Durante la década de los setentas, como respuesta a las condiciones de pobreza en los asentamientos campesinos formados por el Instituto de Tierras y Colonización (ITCO), esta institución puso en marcha una serie de acciones en infraestructura, salud, educación, asistencia técnica, vivienda y crédito. Se promovió la formación de empresas comunitarias de autogestión, principalmente cooperativas. En cuanto al sector cooperativista, la mujer jugó un papel importante, sobre todo aquellas con experiencia en organizaciones sociales, como una forma de encarar el impacto de la crisis económica que inicia a finales de los setentas.

Sin embargo, cabe señalar dos aspectos sobre la participación de las mujeres en las cooperativas. En primer lugar, si bien las mu- 
jeres formaron parte de las cooperativas desde su creación, entre 1979 y 1998 no representaron más del 30\% de los cargos del plenario del Consejo Nacional de Cooperativas y es hasta 1999 que alcanzaron el 37,9\%. En segundo lugar, la participación de mujeres cooperativistas en las zonas rurales se registró mayoritariamente en aquellas cooperativas de crédito y ahorro o ligadas al sector de servicios, no en cooperativas de actividades agropecuarias. En las pocas cooperativas agrícolas en donde se identifica la participación de la mujer durante la década de los ochentas, ésta tenía que ver con la resolución de necesidades básicas asociadas a la inminente crisis económica, y no a un cambio en la cultura campesina respecto al género. Por ejemplo, en el caso de las cooperativas cafetaleras, muchas mujeres se inscribían y recibían crédito, pero este dinero no era necesariamente controlado por ellas sino por sus maridos (Mora, 2013).

\section{Participación en la fuerza laboral}

Las mujeres fueron incorporadas a la economía principalmente como "colchón de la crisis". En términos de León (1993), en este período las mujeres en América Latina cumplieron una "función de amortiguamiento", la cual, con la extensión del trabajo productivo y reproductivo, podría de una u otra forma compensar las restricciones fiscales del modelo económico. De manera que "el papel que la mujer cumple en la sobrevivencia se define como un componente fundamental en la deuda social" (p. 42).

Para el caso costarricense, en 1984 la Fundación Nacional de Clubes 4 S estableció una alianza con el Instituto Nacional de Aprendizaje (INA) y el Ministerio de Educación Pública (MEP) para crear el Programa de Maquila "Mujeres en Acción". Este programa promovió la incorporación de los clubes rurales femeninos a la confección de uniformes escolares para los estudiantes de primaria de todo el país, como un trabajo remunerado. Se registraron aproximadamente 65 grupos de mujeres con una producción de 40000 uniformes al año. Sin embargo, las mujeres no contaban con un salario fijo, sino que recibían una baja paga por cada pieza terminada y, además, sus contratos eran intermitentes o temporales (Baldizán y Cruz, 1987). La entrada de las mujeres al mercado laboral es lo que se conoce como el fenómeno de "feminización del trabajo", 
caracterizado por funcionar en condiciones de incumplimiento de derechos laborales, tanto en el sector agrícola, como el de industria y servicios. Cabe destacar que para $1984 \mathrm{el} 60 \%$ de las mujeres asalariadas recibían menos que el salario mínimo (Madden, 1992).

\section{De amas de casa a protagonistas del desarrollo (1990 a la actualidad)}

La década de los noventas fue un momento histórico clave en materia de derechos para las mujeres costarricenses y con consecuencias particulares en cuanto derechos sobre la tierra. En 1990 se aprobó la Ley de Promoción de Igualdad Social de la Mujer ( ${ }^{\circ} 7142$ ), el cual representa un instrumento esencial en cuanto al avance en igualdad de derechos para las mujeres. Tras la promulgación de dicha ley, se pusieron en práctica una serie de acciones dirigidas a incorporar el enfoque de género en el sector agropecuario. En esta sección se presentan algunos de estas acciones, las cuales se componen de instrumentos legales, políticas, planes y oficinas sectoriales.

\section{Instrumentos legales}

La Ley $\mathrm{N}^{\circ} 7142$ es en buena parte producto de las presiones generadas por los movimientos feministas que se conformaron desde los años setenta. Asimismo, para el tema del acceso a la tierra, el rol de las funcionarias públicas fue clave, en cuanto ellas comenzaron a generar discusiones a lo interno del Instituto de Desarrollo Rural (IDA) y el MAG para mejorar las condiciones de vida de las mujeres rurales. Por ejemplo, en 1984 funcionarias del IDA y del MIDEPLAN llevaron a cabo un primer espacio de discusión institucional sobre la situación de la mujer rural, titulado "Taller Nacional sobre la participación de la mujer campesina en actividades productivas del sector primario" en donde se debatió sobre la discriminación legal y la reproducción de los patrones culturales que han limitado el acceso de las mujeres a la tierra (Madden, 1992). Durante este mismo período, las funcionarias encargadas de coordinar los Clubes $4 \mathrm{~S}$ de mujeres, comenzaron a presionar respecto a la necesidad de mayor presupuesto, para que todos los clubes pudiesen desarrollar proyectos productivos y que las manualidades pudiesen ser comercializadas por las mujeres. Sin embargo, estas demandas no fueron 
acogidas por los funcionarios en la directiva de la institución y el programa no obtuvo mayor apoyo financiero ni operativo (Baldizán y Cruz, 1987).

Años más tarde, la promoción de la Ley $N^{\circ} 7142$ significó un hito a nivel latinoamericano en cuanto al acceso de las mujeres a la tierra, presentando un articulado controversial que posteriormente fue sujeto de cambios. Este instrumento estipuló una medida legal concreta para garantizar, por un lado, la igualdad de condiciones, y por otro lado, una discriminación positiva en favor de las mujeres. Concretamente, se estableció la obligación de que toda propiedad inmueble (tierra y vivienda) otorgada mediante programas de desarrollo social, debía inscribirse a nombre de ambos cónyuges en caso de matrimonio, y a nombre de las mujeres en caso de unión de hecho. En otras palabras, la normativa planteó la posibilidad de co-propiedad en el caso de parejas casadas y la prioridad de titulación femenina en caso de las parejas convivientes. Según explican Deere y León (2000), esta medida de discriminación positiva se contempló con base en "la desprotección que tradicionalmente han sufrido las mujeres en uniones de hecho, y en la importancia de dotar al núcleo familiar de continuidad, estabilidad y cohesión" ante la posibilidad del abandono del marido (p. 249).

La controversia suscitada por dicha medida en favor de las mujeres puede ser explicada por varios factores. En primer lugar, la ley no fue acompañada de un proceso integral de acceso a herramientas educativas que permitieran a las mujeres conocer sobre sus derechos y en muchas ocasiones, se les adjudicó tierra sin ni siquiera haberla solicitado ante el IDA. Esto generó conflictos en los núcleos familiares y representó nuevas cargas de trabajo a las mujeres que no necesariamente habían recibido el adecuado acompañamiento institucional para afrontarlo. En segundo lugar, para los campesinos la ley tampoco resultó agradable y esto quedó plasmado en una demanda presentada por un grupo de hombres de un asentamiento campesino en la Región Huetar Norte. Los querellantes alegaron que el articulado respecto al caso de las uniones de hecho era inconstitucional, pues les discriminaba. El caso llegó hasta la Corte Suprema de Justicia, la cual falló a favor de los campesinos y declaró que también en las uniones de hecho las adjudicaciones de tierra deben titularse a nombre de ambos conyugues (Deere y León, 2000). 


\section{Políticas y planes sectoriales}

La Administración de 1994-1998 puso en marcha el enfoque "Género, Medio Ambiente y Desarrollo Sostenible", en tono con las múltiples conferencias internacionales que apuntaban a incorporar a las mujeres como protagonistas de modelos de desarrollo sostenible. A partir de dicho enfoque se crearon programas y se generaron acciones para mejorar las condiciones de vida de las mujeres rurales (Chinchilla et al., 2000). Más adelante, durante la Administración de 1998-2002, el planteamiento de "Mujer, Desarrollo y Medio Ambiente" fue absorbido por el enfoque de "Desarrollo Humano". En el plano discursivo, se planteó un cambio de jerga institucional de mujer a género y de derechos de las mujeres a derechos humanos. En el plano de la política pública, se implementó un plan sectorial de género para el período de 1999 a 2002. Más adelante, la Administración 2002-2006 puso en marcha una política de género y un plan de acción estratégico para el sector agropecuario, tomando como antecedentes los instrumentos anteriores. Finalmente, la política pública más reciente es la implementación de un plan sectorial a mediano plazo -a diferencia de los anteriores que cubrían únicamente períodos de gobierno-. Dicho plan contempla género y juventud rural para el período de 2010 al 2021. La Tabla 3 resume las principales políticas públicas con enfoque de género para el sector agropecuario entre 1994 y 2015.

Tabla 3. Políticas públicas para la inclusión de enfoque de género en el Sector Agropecuario (1994-2015)

\begin{tabular}{ccc}
\hline Año & Política pública & Descripción \\
\hline & Programa de & Se crean oficinas de la mujer en todas las insti- \\
& Coordinación \\
de las Oficinas & tuciones, concretamente interesa las creadas en \\
Ministeriales y & MAG, CNP, IDA y MINAE con el propósito de \\
Sectoriales de la & incorporar la perspectiva de género en el quehacer \\
Mujer & & institucional.
\end{tabular}


Programa Mujer y Familia del

1994 Instituto de Desarrollo Agrario

1995

1996

1997

1999
Eje Mujeres del Plan Nacional de Combate a la Pobreza

Creación de la Asociación Nacional de Mujeres Campesinas

\section{Addendum de}

los Sectores Agropecuarios y del Ambiente al Plan Nacional para la Igualdad de Oportunidades entre Mujeres y Hombres

(PIOMH)

Plan de Acción Sectorial de Género 1999-2002
La Sección Mujer y Familia existía desde 1979, sin embargo, su trabajo no estaba formalizado. Tuvo el propósito de implementar formas de acceso y control de la tierra equitativos, implementar sistemas de titulación que posibiliten el acceso a la tierra a las mujeres jefas de familia y a campesinas bajo la línea de pobreza, incentivar y fortalecer las organizaciones de productoras y grupos mixtos con fines autogestionarios en los asentamientos campesinos, promover sistemas de administración racional de las tierras en los asentamientos campesinos.

Se plantea coordinar con el IDA para la incorporación de las mujeres rurales, mediante cuotas, al programa de Adjudicación de Tierras, en los paquetes integrales de apoyo, los Proyectos de Desarrollo Integral y el Programa de Crédito no convencional.

Nació como resultado de la primera reunión de Mujeres Campesinas en la Agricultura Orgánica, fue impulsada por la Oficina de la Primera Dama y la FAO para promover la concientización sobre el derecho de las mujeres a la tierra. Participó en la Elaboración del Addedum de Ambiente al PIOMH.

Planteó mejorar mecanismos institucionales para la igualdad de oportunidades, reconocimiento del aporte femenino en el campo, fortalecer su competitividad, favorecer inserción de mujeres campesinas en actividades de seguridad alimentaria y generación de valor agregado, coadyuvar al empoderamiento de las mujeres como "agentes dinamizadoras del desarrollo", incorporar la visión de género en los lineamientos y metodologías de trabajo para la transferencia tecnológica e investigación, promover mayor participación de las mujeres en los sistemas de producción en fincas e incorporar enfoque de género en Sector Ambiente.

Forma parte de las Políticas Públicas para el Sector Agropecuario 1999-2002. Plantea la coordinación sectorial de género a cargo de las Oficinas de Género y Desarrollo (MAG, IDA, CNP, PIMA, SENARA, INCOPESCA) que se encargan de coordinar, monitorear y evaluar las acciones sectoriales de incorporación del enfoque de género en relación con el Programa de Desarrollo Rural. Nace ante la necesidad de impulsar acciones estratégicas encaminadas a la reducción de brechas de género, principalmente relacionadas con aspectos productivos y económicos en el sector rural costarricense. 
2003

Política de Género para el Sector Agropecuario

Costarricense 2002-2010

Plan Sectorial de Género y

2011 Juventud Rural 2010-2021

Eje estratégico "Equidad e inclusión de la población" en

2015 la Política de Estado para el Desarrollo de los Territorios Rurales 2015-2030
Plantea el fortalecimiento de unidades de género en el sector; transversalización de la perspectiva de género en competitividad, capacidades humanas, agricultura sostenible, seguridad alimentaria y modernización institucional; capacitación al personal de las instituciones.

Nace en el marco de la Política de Estado para el Sector Agroalimentario y el Desarrollo Rural

Costarricense 2010-2021. Plantea desarrollar cuatro componentes: desarrollo de capacidades a las mujeres, personas jóvenes rurales y personas técnicas del sector agropecuario; adecuación de servicios institucionales; articulación y fomento de la asociatividad; y gestión del conocimiento con enfoques de género y juventud; componentes que se detallan a continuación.

En esta sección, la situación de las mujeres rurales se trabaja de manera conjunta con la de personas con discapacidad, adultos mayores, diversidad cultural y juventud. La política incorpora un cambio de enfoque en el que se establece el "territorio rural" como base de la planificación. Un aspecto importante de este enfoque es que resalta la necesidad de garantizar el acceso de las mujeres a los activos productivos, así como el fortalecimiento de la participación de las mujeres en el diseño y ejecución de planes de desarrollo a nivel local.

Fuente: Elaboración propia con base en Chinchilla, Hernández y Piedra (2000); Deere y León (2000); INDER (2018); SEPSA (2012); Zumbado (2003).

\section{Oficinas sectoriales}

En 1994 se crearon las Oficinas Ministeriales y Sectoriales de la Mujer en los ministerios del gobierno nacional, coordinadas a cargo del Centro Nacional para el Desarrollo de la Mujer y la Familia, institución antecesora del Instituto Nacional de la Mujer (INAMU). Siguiendo dicha iniciativa, en este mismo año el entonces IDA formalizó el Programa Mujer y Familia, con el objetivo de brindar apoyo a las mujeres rurales en el acceso a la tierra. Dicho programa fue vigente hasta el 2012, ya que con el cambio del IDA al INDER, las funciones se dividieron en diferentes oficinas a lo interno del organigrama institucional.

En 1997, se creó la Unidad Sectorial de Género y Desarrollo en la Secretaría de Planificación Sectorial Agropecuaria (SEPSA), con 
el objetivo de contribuir a la institucionalización del enfoque de género en las instituciones que componen el sector agropecuario. La coordinación del sector se hizo a través del nombramiento de "enlaces de género" en las instituciones. Se trata de funcionarias y funcionarias quienes asumirían dichas labores por recargo, ante la falta de recurso humano. Más tarde, mediante dicha Unidad Sectorial se formuló el Plan de Acción Sectorial de Género. Por medio de este plan se desarrollaron acciones de capacitación, servicios de apoyo, financiamiento, proyectos productivos e información y comunicación (SEPSA, 2003).

En 2010 la Unidad Sectorial se transformó en la Red Sectorial de Género y Juventud Rural ${ }^{3}$, en seguimiento al Plan Sectorial de Género y Juventud Rural 2011-2021. Se trata de un espacio de coordinación interinstitucional para promover el enfoque de género en los servicios brindados por las instituciones del sector agropecuario. Esto se hace mediante asesoría y asistencia técnica a las diferentes dependencias a lo interno de las instituciones. Además de colaborar a lo interno del Sector Agropecuario, la Red coordina con el INAMU y la FAO (INTA, 2017).

\section{MUJERES EN EL DESARROLLO SOSTENIBLE ¿SALTO CUALITATIVO O ELEMENTO DISCURSIVO?}

Resulta evidente que la posición de las mujeres frente a la política pública en materia agropecuaria ha sufrido ciertos cambios a lo largo del tiempo. Para el período que va desde 1950 hasta 1990, las mujeres fueron consideradas como sujetos pasivos del desarrollo agropecuario, incluidas subordinadamente en su rol de amas de casa y esposas. A partir de 1990, se visualiza una transformación relativa en cuanto a su rol en el agro. En tono con las conferencias internacionales, las mujeres en la política pública pasaron de ser ayudantes de los agricultores a protagonistas de la promesa de un desarrollo sostenible. Esto se visualiza en las acciones emprendidas

3 La Red está conformada por el MAG, el Servicio Fitosanitario (SFE), el Servicio Nacional de Salud Animal (SENASA), el Consejo Nacional de Clubes $4 \mathrm{~S}$ (CONAC), el INDER, el Instituto Costarricense de Pesca y Acuicultura (INCOPESCA), el Consejo Nacional de Producción (CNP), el Instituto de Innovación y Transferencia en Tecnología Agropecuaria (INTA), el Programa de Mercadeo Agropecuario (PIMA), la Oficina Nacional de Semillas (ONS), el Servicio Nacional de Aguas Subterráneas (SENARA) y SEPSA. 
con el objetivo de incorporar la perspectiva de género en el sector agropecuario. Sin embargo, lejos de un salto cualitativo en cuanto la condición de las mujeres rurales frente al acceso, uso y control de la tierra, la incorporación del enfoque de género ha sido predominantemente discursivo. En los siguientes párrafos se presentan algunas de los argumentos que pretenden sustentar dicha afirmación.

\section{Ampliación de derechos en espacios reducidos}

Se evidencia la tendencia latinoamericana mencionada por León (1993) en cuanto a políticas públicas en materia del agro. Durante la década de los noventa, hubo una significativa ampliación de los derechos de las mujeres en diferentes espacios del desarrollo de los países, pero, paradójicamente, se redujo al mismo tiempo el contenido de dichos espacios. En otras palabras, se implementaron acciones para mejorar la condición de vida de las mujeres, pero esto ocurre en un momento histórico en el que la función social del Estado es cada vez más limitada. Concretamente en cuanto al agro, si bien se evidencia una clara ampliación de los derechos de las mujeres frente a la tierra, al mismo tiempo se profundiza en un modelo de desarrollo que apunta cada vez más hacia los monocultivos de exportación, dando la espalda a la agricultura familiar y campesina. Por ejemplo, esto se ve reflejado en el estudio de Bonilla (2010), quien concluye que, en la zona sur del país, las mujeres son afectadas diferenciadamente por la concentración de tierras en manos de piñeras.

Podemos ver esta ampliación de derechos y una paralela reducción de espacios a partir de los datos de adjudicación de tierras por parte del IDA. La Figura 1 muestra que, durante dos de tres años en que estuvo vigente la Ley $\mathrm{N}^{\circ} 7142$ sin modificación (1990-1993) ${ }^{4}$, el porcentaje de mujeres beneficiarias de la adjudicación de tierras del IDA aumentó significativamente respecto a las décadas anteriores. Sin embargo, resulta esencial complementar estos datos para tener una perspectiva general del panorama.

\footnotetext{
${ }^{4}$ Como resultado de la resolución de la Corte Suprema de Justicia sobre la querella presentada, la adjudicación conjunta de las uniones de hecho inició hasta 1994 (Deere y León, 2000).
} 
Figura 1. Adjudicaciones de tierra por el IDA según sexo, 1986-1992

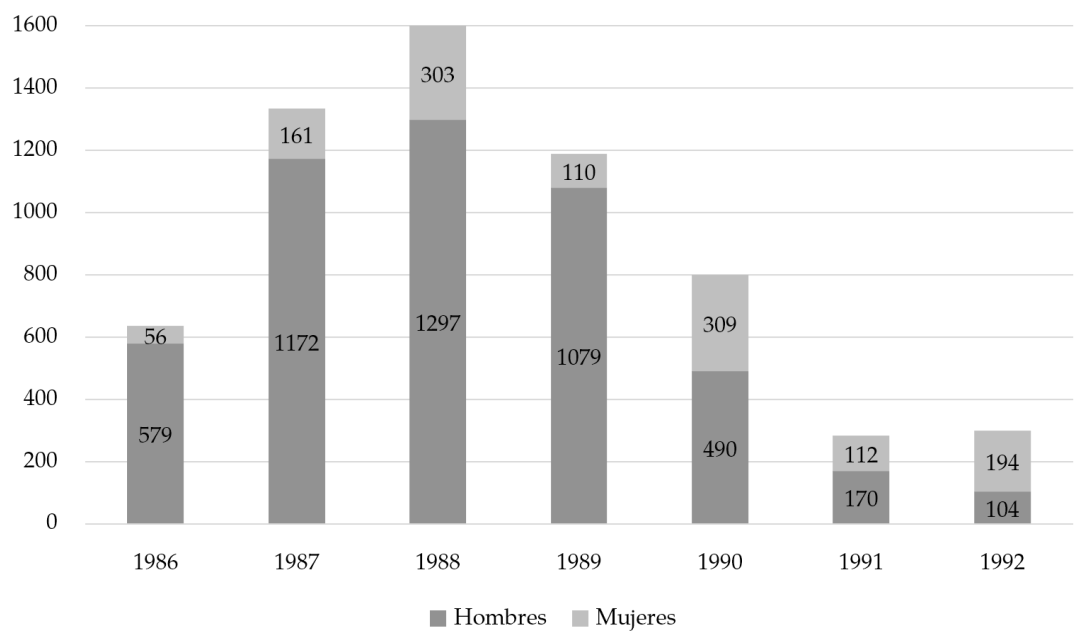

Fuente: Elaboración propia con base en Deere y León (2000).

Para el período de 1998 al 2001, efectivamente la brecha en adjudicaciones de tierra se redujo significativamente, con un $26 \%$ de las parcelas otorgadas a hombres, $25,4 \%$ a mujeres y $48,6 \%$ a parejas. Sin embargo, no se observan los mismos resultados en cuanto a otros insumos asociados al acceso a la tierra. Por ejemplo, para el 2001 se registra que solamente el 12\% de los servicios de extensión agropecuaria fueron otorgados a mujeres. Para el 2003, solamente el 8,7\% de los proyectos de pequeña empresa agropecuaria del Banco Nacional fueron entregados a mujeres. No obstante, se observa una excepción para el caso del programa de proyectos productivos agropecuarios del IDA, llamado PROINDER, en donde los subsidios entregados fueron menos desiguales en términos de género (SEPSA, 2003).

De manera tal que, los datos presentados, si bien sumamente generales, permiten dar cuenta de una contradicción en cuanto a las mujeres frente a la política pública agropecuaria. Efectivamente, la brecha en cuanto a la adjudicación de tierras se ha disminuido considerablemente, pero esto no ha sido igual para otros servicios agropecuarios asociados al acceso a la tierra. Además, ocurre una ampliación de los derechos de las mujeres en el espacio agropecuario al tiempo que se reduce el contenido de este espacio, como resultado de la transformación en la matriz productiva. Aunado a 
esto, cabe destacar que desde hace más de sesenta años una gran cantidad de asentamientos campesinos conformados por el ITCO-IDA sufren condiciones de inseguridad jurídica debido a falta de una titulación formal sobre la tierra (Barquero, 2018). Dicho desorden institucional es un reflejo de la posición marginal que ocupa el campesinado dentro de la política pública agropecuaria, a pesar de su importancia para el desarrollo sostenible. Dentro de esta posición marginal, las mujeres presentan mayores dificultades para ejercer plenamente los derechos de tenencia, uso y control de la tierra.

\section{Los efectos de la reconversión productiva sobre las mujeres}

El discurso del desarrollo sostenible se mostró versátil para incluir el tema de género y de forma paralela, para hacerlo compatible con el proceso de la transformación del agro. La Administración 19941998 puso en marcha el Programa de Reconversión Productiva del Sector Agropecuario, mediante la cual se profundizó el modelo de desarrollo iniciado desde la firma de los Programas de Ajuste Estructural durante los ochentas. El objetivo principal de la reconversión productiva fue de reorientar la economía hacia la producción de productos de mayor valor agregado, principalmente aquellos dedicados a la exportación.

Las mujeres rurales fueron incluidas en los proyectos desarrollados en el marco de la reconversión productiva, en conjunto con las demás acciones institucionales mencionadas. A pesar de ello, la brecha continuó siendo sumamente amplia para el período de 1996 a 2002, durante el cual solamente un 5,1\% de los proyectos de reconversión productiva contaron con participación femenina. No obstante, se realizaron esfuerzos por ejecutar programas de capacitación para mujeres rurales y se aprobó un proyecto de desarrollo microempresarial. En términos generales, se visualizan avances para posicionar a las mujeres dentro del desarrollo agropecuario mediante la política pública (SEPSA, 2003).

A nivel discursivo, se argumentó que la reconversión productiva sería sumamente beneficiosa para las mujeres rurales, quienes verían aumentadas sus posibilidades de acceder a crédito, asistencia y capacitación, así como a trabajo agrícola asalariado. Sin embargo, si la reconversión productiva presenta verdaderamente 
un escenario beneficioso para las mujeres, es sumamente debatible. En la práctica, la transformación del agro vino a cambiar radicalmente la estructura productiva del país, beneficiando al gran sector agroexportador, en detrimento de la agricultura campesina. Esto tiene consecuencias específicas sobre las mujeres, ya que ellas históricamente predominan en la agricultura de subsistencia y producción de alimentos para el consumo nacional. Al igual que en otros países de América Latina, la reconversión productiva ha provocado una asalarización de las mujeres en labores agrícolas. Dicha feminización del agro, lejos de contribuir a su autonomía económica, presenta condiciones de extrema flexibilidad y explotación laboral. Las mujeres son contratadas en labores que reproducen sesgos de género, ya que los empleadores apelan a una supuesta manipulación más cuidadosa de productos perecederos como las flores (Kay, 2007).

En Costa Rica, las mujeres se han incorporado a la fuerza laboral agrícola mayoritariamente en labores de procesamiento industrial (corte, lavado y empaque), principalmente en frutas y hortalizas. La mayoría de ellas son contratadas de forma temporal y con altos grados de informalidad. En muchas ocasiones, sus remuneraciones no cumplen con el salario mínimo ya que se trabaja "a destajo", lo cual asegura ciertos niveles de productividad para las empresas, pero no garantiza salarios justos para las trabajadoras. Asimismo, se presenta un profundo incumplimiento de derechos laborales mínimos. En dichas condiciones, el proceso de asalarización no logra contribuir a la superación de la pobreza de las mujeres y sus familias, si no que perpetúa las condiciones de vulnerabilidad ante la carencia de empleo digno y formal y desmantelamiento de la agricultura campesina (Subercaseaux, 2015).

\section{DESARROLLO TERRITORIAL, ¿OPORTUNIDAD O DESAFÍO PARA INCLUIR A LAS MUJERES?}

El cambio institucional del IDA al INDER implicó una transformación significativa en términos de óptica. En el marco del desarrollo sostenible, se puso en marcha el enfoque de la nueva ruralidad, mediante una estrategia de desarrollo rural territorial. Bajo esta nueva visión, se establece el territorio rural como elemento base de la planificación y operación de las políticas públicas, desde una perspectiva amplia de la ruralidad, con el fin de lograr una mejor 
gobernanza territorial. Desde dicha posición, se argumenta que es prioritario garantizar el acceso de las mujeres a los activos productivos, tales como tierra, recursos naturales y acceso al crédito, así como el fortalecimiento de la participación de las mujeres en el diseño y ejecución de planes de desarrollo a nivel local (Inder, 2018).

Sin embargo, existen posturas críticas frente a la óptica de la nueva ruralidad, principalmente en cuanto a su incorporación en las políticas para las zonas rurales. Kay (2009) señala que la nueva ruralidad "se ha convertido en un concepto paraguas utilizado para referirse a cualquier nuevo desarrollo en las áreas rurales, o cualquier problema relegado al que no se le prestara suficiente atención en esquemas de trabajo anteriores" (p. 610). Evidentemente, esto no resulta en algo negativo per se; sin embargo, en muchas ocasiones, si bien se visibiliza la situación de las poblaciones vulnerables en el campo, se profundiza poco sobre las condiciones de clase que determinan dicha vulnerabilidad. Esto ocurre porque el enfoque de la nueva ruralidad pretende conciliar los diferentes actores y visiones que existen en los territorios rurales, prestando poca o nula atención a las tensiones y conflictos que se generan a partir de ellas. Así, se idealiza una convivencia relativamente armónica entre grandes cadenas agroproductivas y agriculturas campesinas de subsistencia, al tiempo que se fomenta la participación de sectores históricamente olvidados.

Los datos presentados permiten argumentar que la agricultura de gran escala ha crecido a costa de la agricultura campesina. De manera que, en dicho cometido de inclusión en el plano discursivo, la política agropecuaria tiene el desafío de incorporar el lenguaje de género sin perder su potencial transformador, tal como ha señalado Deere (1993). Por un lado, resulta contradictorio destacar el rol de las mujeres al tiempo que se han ido desmantelando los espacios en los que históricamente su participación ha sido crucial. Por otro lado, el hecho de que la planificación de género se realice de forma paralela a la de otras poblaciones vulnerables como por ejemplo jóvenes, personas con discapacidad y minorías étnicas, puede resultar en una limitada comprensión de las necesidades específicas de cada población.

En el plano operativo, uno de los hallazgos a partir de las entrevistas realizadas en el marco de la Red Sectorial de Género es que la institucionalización del enfoque de género es uno de los mayores desafíos del sector. Si bien actualmente existen esfuerzos por sen- 
sibilizar a las y los funcionarios, aún persisten grandes retos para que esto se traduzca con eficacia en el accionar institucional cotidiano. Por ejemplo, al trabajar en proyectos productivos con mujeres, resulta necesario tomar en cuenta las condiciones específicas de género que pueden afectar la productividad de las fincas, lo cual repercute en cumplimiento de los estándares productivos esperados por la institución. Además, otro elemento clave para la institucionalización del enfoque es la creación de cargos con presupuesto propio -y no por recargo, como es actualmente- para trabajar el tema de género y cumplir tan ambiciosos objetivos de inclusión.

Aunado a esto, resulta necesario analizar críticamente el cambio en el modelo estatal de dotación de tierra y sus repercusiones para el acceso, uso y control de la tierra para las mujeres. Actualmente, la tierra es otorgada por el INDER bajo la forma de arrendamiento agrario. Se trata de un contrato de uso del predio por tiempo limitado, firmado entre la institución y la persona o grupo beneficiario, bajo el cumplimiento de una serie de compromisos de uso y eficiencia. Este cambio busca remediar la irregularidad en el uso de la tierra que caracterizó los modelos anteriores, en donde muchas de las familias beneficiarias incurrieron en prácticas de venta o alquiler de los terrenos otorgados. De manera que, para acceder a la tierra se debe previamente formular un proyecto productivo con indicadores de productividad que serán medidos por la institución, de los cuales dependerá la prolongación del arrendamiento. Puede ser desde un proyecto de agricultura hasta un proyecto de infraestructura, pero debe cumplir con una serie de estándares que garanticen su viabilidad económica y operativa, según lo indica la Ley del INDER 9036 (2012).

Uno de los principales retos que este cambio representa en cuanto al acceso y uso de la tierra es el acompañamiento que el INDER pueda otorgar a las mujeres rurales, tanto para formular un proyecto productivo como para llevarlo a cabo una vez firmado el contrato de arriendo. Este acompañamiento debe necesariamente incorporar el enfoque de género, ya que las condiciones y necesidades de las mujeres campesinas requieren de atención particular con respecto a la de los hombres campesinos. Tal como se ha mencionado a lo largo del artículo, las mujeres tienen menor acceso a recursos productivos, tecnológicos y educativos y además tienen mayores cargas de trabajo doméstico y de cuido. En este sentido, sus herramientas y tiempos no son los mismos y esto repercute en 
sus posibilidades para formular y gestionar proyectos productivos.

Aunado a esto, resulta importante que el acompañamiento sea lo suficientemente participativo para lograr respetar la autonomía campesina, lo cual está relacionado con el control de la tierra para las mujeres. Sin duda alguna, esto representa un gran desafío debido a que, bajo el nuevo modelo, los proyectos productivos priorizan el cultivo mayoritario de un solo producto que tenga buenos resultados en el mercado. Es decir, se limita la autonomía de las personas campesinas de decidir qué sembrar y cómo hacerlo, ya que deben cumplir con los estándares productivos establecidos previamente. El nuevo modelo busca movilizar la tierra como un recurso económico, lo cual puede contribuir a aliviar algunas carencias de las familias campesinas empobrecidas. Sin embargo, en el largo plazo amenaza también su soberanía alimentaria, es decir la capacidad de las comunidades de producir y distribuir localmente sus propios alimentos, adaptados ecológica y culturalmente a su entorno ${ }^{5}$. En un contexto en el que las mujeres tienen grandes dificultades para controlar efectivamente sus predios, presentan mayores niveles de pobreza y menor acceso a recursos básicos, la soberanía alimentaria resulta un aspecto crucial para su supervivencia y la de sus familias.

A pesar de dichos desafíos, cabe resaltar que el enfoque de desarrollo territorial puede ser efectivamente una oportunidad para visibilizar el trabajo de las mujeres en el agro y avanzar hacia su inclusión en la política pública, con miras a disminuir la brecha de género. La posibilidad de financiamiento para proyectos productivos permite promover la participación no solo de familias, sino también de grupos organizados. Esto presenta una oportunidad interesante porque implica un reconocimiento de la propiedad agropecuaria más allá de la unidad productiva familiar, la cual no ha demostrado ser necesariamente el esquema más equitativo para la distribución de la tierra. Esto debido a que la política pública precedente devela un entendimiento limitado de las relaciones de poder a lo interno del hogar, en detrimento del acceso de las mujeres a la tierra. Mientras que, la posibilidad de acceder a la tierra mediante grupos organizados puede venir acompañada de formas más equitativas en cuanto a la toma de decisión sobre el predio y

5 Dicha definición del concepto soberanía alimentaria es basada en la propuesta del movimiento La Vía Campesina, el cual fue públicamente debatido en ocasión de la Cumbre Mundial de la Alimentación en 1996. 
la producción.

\section{CONCLUSIONES}

Al estudiar la brecha de género en el acceso a la tierra, resulta necesario tomar en cuenta tres elementos principalmente. En primer lugar, es fundamental definir el acceso a la tierra desde un enfoque feminista que permita analizar, de manera multidimensional, su relación con la autonomía de las mujeres. Es decir, comprender la tierra más allá de su función como un activo económico, sino como un tejido de relaciones sociales que influyen en la vida cotidiana de las mujeres, sus familias y sus comunidades. Por esto, es preciso definir el acceso como un conjunto de derechos relacionados con la tenencia, el uso y el control.

En segundo lugar, uno de los principales desafíos en materia de política pública es invertir en investigación que permita al sector conocer las necesidades de las mujeres rurales en cuanto al acceso a la tierra. Para este cometido, la perspectiva de género en la formulación, aplicación y análisis en los censos agropecuarios resulta esencial. En tercer lugar, resulta necesario contextualizar la brecha de género en los esquemas más amplios de distribución de la tierra, ya que esto revela una serie de mecanismos estructurales que afectan diferenciadamente a las mujeres.

Precisamente, al analizar el contexto más amplio de desigualdad en el acceso a la tierra, se hace evidente que existe una profunda contradicción entre la promoción del desarrollo sostenible y la paralela desmantelación de la agricultura campesina. Si bien las mujeres se han beneficiado de dicho cambio de enfoque al ser mayormente visibilizadas en la política pública, al mismo tiempo los espacios de su participación se han visto amenazados por la reconversión productiva. Históricamente, las mujeres han jugado un papel central en la producción de alimentos básicos, en las huertas familiares y cuido de animales. Todos estos son espacios productivos que actualmente ocupan una estructura marginal dentro de una política agropecuaria dirigida principalmente a la producción a gran escala para la exportación.

Por su parte, el más reciente enfoque de desarrollo rural territorial presenta una ambiciosa agenda para incluir a las mujeres rurales. En cuanto a ello, uno de los principales retos es que el enfoque 
de género sea efectivamente institucionalizado. Para ello, se requiere mejorar las capacidades operativas de los enlaces de género dentro del sector agropecuario. Asimismo, el enfoque territorial se presenta como una oportunidad para la promoción de la participación de las mujeres en los espacios rurales. Esto debido a que logra trascender el modelo de dotación de tierras basado en unidades productivas familiares, entendidas desde una visión tradicionalmente patriarcal, con los hombres como jefes de producción y las mujeres como ayudantes.

De manera que, institucionalizar el enfoque de género implica su puesta en práctica en toda la amplia gama de servicios que ofrece el sector agropecuario: adjudicación de tierras, asistencia técnico-financiera, extensión agropecuaria, acceso a crédito, entre otros. Asimismo, implica poner el lente de género en la formulación, aplicación y análisis de los censos agropecuarios. Para lograr este cometido, es necesario mejorar la capacidad operativa de los enlaces de género en todas las instituciones del sector. Esto podría además estar relacionado con la representación de mujeres en el organigrama del sector agropecuario, el cual continúa hoy siendo sumamente masculinizado.

\section{REFERENCIAS}

Agarwal, B. (1994). A field of one's own. Gender and land rights in South Asia. Cambridge University Press.

Agarwal, B. (1997). Bargaining and gender relations: Within and beyond the household. Feminist Economics, 3 (1), 1-51.

Baldizán, G., y Cruz, F. (1987). La Mujer Rural en los Grupos Productivos y la Politica Estatal de Apoyo (El Caso del Programa Clubes 4-S y Amas de Casa) [Tesis para optar por el grado de Licenciatura en Sociología]. Universidad de Costa Rica.

Barquero, M. (2018, julio 17). Inder acumula desorden de 60 años con tierras sin regular. La Nación, Grupo Nación. https://cutt.ly/rgYvcNS

Blanco, L. (1997). Las políticas de tierra en Centroamérica: Una visión desde las mujeres. Perspectivas Rurales Nueva Época, 0(2). http://www. revistas.una.ac.cr/index.php/perspectivasrurales/article/view/3405

Bonilla, A. (2010). Más desposeídas que propietarias: El acceso a la tierra, también una cuestión de género. Fondo de Desarrollo de las Naciones Unidas para la Mujer (UNIFEM). 
Bórquez, R., y Ardito, L. (2009). Experiencias activas de acceso a la tierra: Estrategias de empoderamiento y aseguramiento de derechos desarrolladas por organizaciones de mujeres campesinas e indígenas rurales [Informe de investigación]. International Land Coalition América Latina. http:// americalatinagenera.org/newsite/images/experiencias_activas_de_ acceso_a_la_tierra.pdf

Castillo, A. (2015). Las mujeres y la tierra en Guatemala: Entre el colonialismo y el mercado neoliberal. Serviprensa.

Chinchilla, M., Hernández, N., y Piedra, M. (2000). La reconversión productiva del sector agropecuario en Costa Rica: La reforma en el contexto del desarrollo sostenible y su formulación en la perspectiva de género: 1996-1999. [Tesis para optar por el grado de Licenciatura en Ciencias Políticas]. Universidad de Costa Rica.

Deere, C. (1987). The Latin American Agrarian Reform Experience. En Rural Women and State Policy: Feminist perspectives on Latin American Agricultural Development. Westview Press.

Deere, C., y León, M. (2000). Género, propiedad y empoderamiento; tierra, Estado y mercado en América Latina. Tercer Mundo Editores.

FAO. (2011). El Estado Mundial de la Agricultura y la Alimentación. Oficina de Intercambio de Conocimientos, Investigación y Extensión, FAO. http://www.fao.org/3/a-i2050s.pdf

Fuentes, A. (2010). Mujeres rurales, tierra y producción: Propiedad, acceso y control de la tierra para las mujeres (E. Campbell, Ed.). http://genderandsecurity.org/sites/default/files/Fuentes_Lopez_-_MjR_tt_produccn.pdf

Golen, B., y Medina, M. (2017). Características de la participación agropecuaria de las mujeres según datos del CENAGRO 2014. Una visión del sector agropecuario basada en el CENAGRO 2014, San José, C.R.

Guillamón, A., y Ruíz, C. (2015). Feminismos y lucha por el territorio en América Latina. Revista Pueblos, 64. http://www.revistapueblos. org/blog/2015/02/09/feminismos-y-lucha-por-el-territorio-en-america-latina/

Inder. (2018). ITCO-IDA-INDER: Una experiencia con historia.

INEC. (2015). VI Censo Nacional Agropecuario 2014. Instituto de Estadística y Censos. http://www.mag.go.cr/bibliotecavirtual/U40-10581.pdf

Kay, C. (1995). Desarrollo rural y cuestiones agrarias en la América Latina contemporánea. Agricultura y sociedad, 75, 27-82.

Kay, C. (2007). Algunas reflexiones sobre los estudios rurales en América Latina 
(Dossier). http:/ / repositorio.flacsoandes.edu.ec/handle/10469/600

Kay, C. (2009). Estudios rurales en América Latina en el periodo de globalización neoliberal: ¿una nueva ruralidad? Revista mexicana de sociología, 71(4), 607-645.

Korol, C. (2016). Somos tierra, semilla, rebeldía: Mujeres, tierra y territorios en América Latina. América Libre. https://www.grain.org/es/article/ entries/5563-somos-tierra-semilla-rebeldia-mujeres-tierra-y-territorios-en-america-latina

Lastarria-Cornhiel, S., Ranaboldo, C. y Deere, C. (2011). Tierra de mujeres: Reflexiones sobre el acceso de las mujeres rurales a la tierra en América Latina. Fundación TIERRA, Coalición Internacional para el Acceso a la Tierra. http://www.rimisp.org/wp-content/files_mf/1377805458tierramujeresreflexionesaccesotierraenamericalatina.pdf

León, A. (2015). Desarrollo geográfico desigual en Costa Rica. El ajuste estructural visto desde la Región Huetar Norte (1985-2005). Editorial Universidad de Costa Rica.

León, M. (1993). El género en la política pública de América Latina: Neutralidad y distensión. 14.

Ley de Tierras y Colonización (ITCO INDER), Pub. L. No. 2825 (1961). https: / cutt.ly/sgYmIoH

Madden, L. (1992). El acceso de la mujer a la tierra en Costa Rica (Fundación Arias para la Paz y el Progreso Humano, Ed.).

Mora, J. (2013). Mujer y Cooperativismo en Costa Rica. Revista Horizontes Cooperativos, 21. https://issuu.com/cenecooprl/docs/revista_horizontes_coop_mujer_y_coo

Morales, N. y Segura, R. (2016). Distribución de la tierra y su relación con la desigualdad social. http://estadonacion.or.cr/files/biblioteca_virtual/022/Equidad/SimposioCENAGRO.pdf

Moser, C. (1999). Mainstreaming Gender and Development in the World Bank.

ONU. (2015). FAO aboga por mayor acceso de las mujeres a la tierra en América Latina y el Caribe. https://cutt.ly/bgYncJq

Programa Estado de la Nación. (2013). Agricultura y sostenibilidad. (Vigésimo Informe Estado de la Nación). http:/ / estadonacion.or.cr/files/ biblioteca_virtual/020/ambiente/Bach_Agricultura.pdf

Román, I. (1997). Tendencias de cambio social en el proceso de modernización agrícola costarricense (1980-1996). Perspectivas Rurales Nueva Época, 1. http://www.revistas.una.ac.cr/index.php/perspectivasrura- 
les/article/view/3389

SEPSA. (2003). Política de Género y Plan de Acción Estratégico 2002-2006. http://www.mag.go.cr/bibliotecavirtual/E14-4835.pdf

SEPSA. (2012). Plan Sectorial Agropecuario de Género y Juventud Rural 20102021

Subercaseaux, X. (2015). Feminización del empleo y trabajo precario en las agriculturas latinoamericanas globalizadas. Cuadernos de Antropología Social, 17.

Zumbado, C. (2003). Género y políticas de desarrollo: La brecha entre el decir y el hacer. Desarrollo rural y políticas agropecuarias en Costa Rica. [Tesis de Doctorado]. Universidad Autónoma de Barcelona.

MARYLAURA ACUÑA ALVARADO es costarricense. Licenciada en Ciencias Políticas por la Universidad de Costa Rica. Estudiante de la Maestría de Titulación Conjunta en Desarrollo Territorial Sostenible de la Universidad de Padua, Universidad Católica de Lovaina y Universidad Paris 1 Panteón-Sorbona. Ha sido asistente en proyectos de investigación del Centro de Investigación y Estudios Políticos (CIEP), el Observatorio del Desarrollo (OdD) y el Programa Kioskos Socioambientales, todos relacionados con temáticas de política ambiental y agropecuaria, regiones transfronterizas, agricultura campesina, trabajo agrícola y organización comunitaria. 